ABX-Verbindungen mit modifizierter $\mathrm{Ni}_{2} \mathrm{In}$-Struktur

ABX-Compounds with a Modified $\mathrm{Ni}_{2} \mathrm{In}$ Structure

Christa Tomuschat und Hans-Uwe Schuster* Institut für Anorganische Chemie

der Universität zu Köln,

Greinstraße 6, D-5000 Köln 41

Z. Naturforsch. 36 b, 1193-1194 (1981); eingegangen am 25. Mai 1981

Ternary Europium Pnictides, 1A-Elements, Crystal and Magnetic Data

Ten new compounds were synthesized. They crystallize with a $\mathrm{Ni}_{2} \mathrm{In}$-type structure (space group $\mathrm{P}_{3} / \mathrm{mmc}^{-} \mathrm{D}_{6 \mathrm{~h}}^{4}$ ). Their magnetic behaviour was investigated in the temperature range from 80 to $290 \mathrm{~K}$.

Ternäre ABX-Verbindungen $(A=2 a-E l e m e n t$ oder Europium, $\mathrm{B}=1 \mathrm{~b}$-Element, $\mathrm{X}=5 \mathrm{~b}$-Element) kristallisieren unter bestimmten Voraussetzungen im hexagonalen $\mathrm{Ni}_{2} \mathrm{In}-\mathrm{Typ}$, wie anhand einer Reihe von Arbeiten gezeigt werden konnte [1-3]. Dabei werden die Strukturen dieser elektrovalent zusammengesetzten $\mathrm{ABX}$-Verbindungen in erster Linie vom Radienverhältnis $r_{A}: r_{B}$ bestimmt, wobei neben der aufgefüllten Blende- und $\mathrm{Cu}_{2} \mathrm{Sb}$-Struktur mit steigendem Radienverhältnis auch Varianten des anti- $\mathrm{PbCl}_{2^{-}}, \mathrm{Fe}_{2} \mathrm{P}$ - und $\mathrm{Ni}_{2} \mathrm{In}$-Typs ausgebildet werden. Weiterhin ergibt sich bei der Zuordnung der Valenzen für das B-Element eine kugelsymmetrische Anordnung der d-Elektronen.

Da das Seltenerd-Element Europium dem Strontium sehr ähnlich ist, konnten auch EuBX-Verbindungen dargestellt werden. Zur Überprüfung der Oxidationsstufe des Europiums wurden magnetische Messungen im Temperaturbereich von $80-290 \mathrm{~K}$ durchgeführt.

\section{Darstellung und Eigenschaften}

Zur Darstellung der Verbindungen wurden die Elementgemenge im Verhältnis 1:1:1 in Korundtiegeln unter Argon als Schutzgas umgesetzt, wobei die Proben nach 12-16stdg. Erhitzen auf $1000^{\circ} \mathrm{C}$ fein zerrieben und anschließend $14 \mathrm{~h}$ lang bei $1100^{\circ} \mathrm{C}$ getempert wurden. Die Präparate fielen als graublaue, spröde Pulver an, die von Luft und Feuchtigkeit nicht angegriffen wurden.

\section{Strukturbestimmung}

Die Verbindungen sind nach Pulveraufnahmen beurteilt isotyp und lassen sich in Analogie zu den

* Sonderdruckanforderungen an Prof. Dr. H.-U. Schuster.

0340-5087/81/0900-1193/\$01.00/0 bereits bearbeiteten Verbindungen mit $\mathrm{Ni}_{2}$ In-Struktur (Raumgruppe $\mathrm{P}_{3} / \mathrm{mmc}$ ) [1-3] hexagonal indizieren. Die Gitterkonstanten der Verbindungen wurden aus Guinier-Aufnahmen mit Silizium als Eichsubstanz ermittelt.

\begin{tabular}{llll}
\hline Verbindung & $a[\mathrm{pm}]$ & $c[\mathrm{pm}]$ & $c / a$ \\
\hline EuCuP & 412,3 & 820,0 & 1,99 \\
EuCuAs $[1]$ & 425,4 & 827,4 & 1,94 \\
EuCuSb & 451,2 & 854,2 & 1,89 \\
EuCuBi & 462,2 & 853,6 & 1,85 \\
EuAgP & 439,5 & 805,7 & 1,83 \\
EuAgAs & 451,6 & 810,7 & 1,80 \\
EuAgSb & 475,5 & 828,3 & 1,74 \\
EuAgBi & 487,7 & 813,9 & 1,67 \\
EuAuP & 431,3 & 825,8 & 1,91 \\
EuAuAs & 444,5 & 828,5 & 1,86 \\
EuAuSb & 466,9 & 848,6 & 1,82 \\
\hline
\end{tabular}

Repräsentativ für die Verbindungsreihe wurde von EuAgAs eine Strukturrechnung durchgeführt. Für die Intensitätsmessungen wurde zur Vermeidung von Absorptionseffekten ein Einkristall geeigneter Abmessungen ausgesucht, der auf einem automatischen Vierkreisdiffraktometer CAD 4 der Firma ENRAF-NONIUS vermessen wurde (Graphitmonochromator, $\mathbf{M o K}_{\mathbf{x}}$-Strahlung, $\omega / \mathbf{2} \vartheta$-scan, $\left.\vartheta \leqslant 40^{\circ}\right)$.

Nach der Datenreduktion standen 76 Reflexe mit $\mathrm{I} \geqslant 2 \sigma(\mathrm{I})$ zur Verfügung. Zur Strukturrechnung wurden das least-squares-Programm ORFLS [4] sowie Programme des X-Ray-72-Systems [5] herangezogen. Mit 2 Formeleinheiten pro Elementarzelle konnte folgende Punktlagenbesetzung in der Raumgruppe Nr. $194 \mathrm{P}_{3} / \mathrm{mmc}^{-} \mathrm{D}_{6 \mathrm{~h}}^{4}$ angenommen werden:

$$
\begin{array}{lllllll}
2 \mathrm{Eu} \text { in (a) } & 0 & 0 & 0 & & \\
2 \mathrm{Ag} \text { in (d) } & 1 / 3 & 2 / 3 & 3 / 4, & 2 / 3 & 1 / 3 & 1 / 4 \\
2 \mathrm{As} \text { in (c) } & 1 / 3 & 2 / 3 & 1 / 4, & 2 / 3 & 1 / 3 & 3 / 4
\end{array}
$$

\begin{tabular}{|c|c|c|c|}
\hline & $\mathbf{E u}\left[\mathrm{pm}^{2}\right]$ & $\mathrm{Ag}\left[\mathrm{pm}^{2}\right]$ & As $\left[\mathrm{pm}^{2}\right]$ \\
\hline $\begin{array}{l}\mathrm{U}_{11}=\mathrm{U}_{22}=2 \mathrm{U}_{12} \\
\mathrm{U}_{33} \\
\mathrm{U}_{13}=\mathrm{U}_{23}=0\end{array}$ & $\begin{array}{r}70 \\
135\end{array}$ & $\begin{array}{r}55 \\
386\end{array}$ & $\begin{array}{l}17 \\
93\end{array}$ \\
\hline
\end{tabular}

Als anisotrope Temperaturfaktoren* wurden folgende Werte erhalten:

* Der anisotrope Temperaturfaktor ist definiert als: exp. - $\left[2 \pi^{2}\left(h^{2} a^{* 2} U_{11}+k^{2} b^{* 2} U_{22}+l^{2} c^{* 2} U_{33}+\right.\right.$ $\left.\left.2 h k a^{*} b^{*} U_{12}+2 h l a^{*} c^{*} U_{13}+2 k l b^{*} c^{*} U_{23}\right)\right]$.

Der R-Wert betrug 0,06 . 


\section{Beschreibung der Struktur}

Die Atomanordnung bei der Verbindung EuAgAs entspricht jener der $\mathrm{Ni}_{2} \mathrm{In}$-Struktur, wobei die NiAtome durch Europium und Silber ersetzt sind. Das Europium ist in planaren, sich jeweils im Abstand von $\mathrm{c} / 2$ wiederholenden Schichten angeordnet. In den Mittelpunkten der dadurch gebildeten trigonalen Prismen befinden sich abwechselnd je ein Agund ein As-Atom. Dadurch werden planare Sechsecknetze gebildet, die so im Abstand von c/2 übereinander geordnet sind, daß ein Ag-Atom über einem As-Atom zu liegen kommt und umgekehrt.

\section{Magnetische Untersuchungen}

Das Europium besetzt in den hier beschriebenen Verbindungen die Positionen des A-Elements, die sonst von Ca und $\mathrm{Sr}$ eingenommen werden.

Um die Zweiwertigkeit des Europiums in den hier vorgestellten Verbindungen zu überprüfen, wurden die Suszeptibilitäten mittels einer FaradayMagnetwaage bestimmt. Die Messungen ergaben, daß innerhalb des Temperaturbereichs von $80-290 \mathrm{~K}$ die Verbindungen paramagnetisch sind und dem
Curie-Weiss-Gesetz folgen. Das jeweils ermittelte magnetische Moment zeigt, daß Europium in sämtlichen Verbindungen als $\mathrm{Eu}^{2+}$ vorliegt:

\begin{tabular}{llr}
\hline & $\mu\left[\mu_{\mathrm{B}}\right]$ & $\theta[\mathrm{K}]$ \\
\hline EuCuP & 7,64 & 41 \\
EuCuAs & 7,67 & 28 \\
EuCuSb & 7,72 & 7 \\
EuCuBi & 7,65 & -13 \\
EuAgP & 7,52 & 20 \\
EuAgAs & 7,45 & 19 \\
EuAgSb & 7,62 & 2 \\
EuAgBi & 7,39 & -4 \\
EuAuP & 7,68 & 22 \\
EuAuAs & 7,57 & 11 \\
EuAuSb & 7,60 & 6 \\
\hline
\end{tabular}

Der theoretische Wert für $\mathrm{Eu}^{2+}$ beträgt 7,94 $\mu_{\mathrm{B}}$.

Für die Förderung unserer Arbeiten danken wir der Deutschen Forschungsgemeinschaft und dem Verband der Chemischen Industrie.
[1] A. Mewis, Z. Naturforsch. 33b, 983 (1978).

[2] A. Mewis u. P. Blumstengel, Z. Naturforsch. 33 b, 671 (1978).

[3] B. Eisenmann, G. Cordier u. H. Schäfer, Z. Naturforsch. 29b, 457 (1974).
[4] W. R. Busing, K. O. Martin u. M. A. Levy, Oak Ridge 1962.

[5] J. M. Steward, G. J. Kruger, H. L. Ammon, C. Dickinson u. R. S. Hall, Report TR-192, University of Maryland, Maryland 1972. 